\title{
RESPONSE OF BARLEY TO NITROGEN FERTILIZATION, FOLIAR APPLICATION OF MICRONUTRIENTS MIXTURE AND CITRIC ACID UNDER CALCAREOUS SOIL
}

\author{
(Received: 13.12.2015)
}

\author{
By \\ M. Z. Shendy, H. A. Goma* and M. E. Nashed* \\ Barley Research Department and ${ }^{*}$ Crop Physiology Research Department, Field Crops Research \\ Institute, Agricultural Research. Center, Giza, Egypt
}

\begin{abstract}
A field trial was conducted at Nubaria Agricultural Research Station, El-Behira Governorate, Egypt, during two successive growing seasons 2012/2013 and 2013/2014. The aim was to study the effect of nitrogen fertilizer rates i. e. $15,30,45$ and $60 \mathrm{~kg} \mathrm{~N} \mathrm{fed}^{-1}$ and foliar spray by micronutrient mixture $(\mathrm{Fe}+\mathrm{Zn}+\mathrm{Mn})$ at concentrations of 0,133 and $200 \mathrm{ppm}$ for each element and citric acid $(0$, 1000 and $1500 \mathrm{ppm}$ ) on barley cultivar Giza 2000. The rate of $60 \mathrm{~kg} \mathrm{~N} \mathrm{fed}^{-1}$ gave the maximum values of plant height, leaf area/ main stem, number of days to 50\% heading, spike length, crop growth rate (CGR) at the first (60-75) DAS and second (75-90 DAS) periods, number of spikes $\mathrm{m}^{-2}$, number of kernels spike ${ }^{-1}, 1000$-kernel weight as well as straw and grain yields, total chlorophyll of leaves at 75 DAS and protein content of grains. Whereas, insignificant differences were observed in grain yield between applying 45 and $60 \mathrm{~kg} \mathrm{~N}$ fed $^{-1}$. All growth traits, yield and its components as well as total chlorophyll of leaf and protein content of grains recorded the maximum values when barley plants were sprayed by $200 \mathrm{ppm}$ of micronutrients mixture $(\mathrm{Fe}+\mathrm{Zn}+\mathrm{Mn})$ for each element plus $1500 \mathrm{ppm}$ citric acid $\left(S_{2}\right)$ compared to other foliar spray treatments $\left(S_{0}\right.$ and $\left.S_{1}\right)$. The interaction between nitrogen fertilizer rates and foliar spray of micronutrients mixture plus citric acid had a significant effect on all traits under study except the number of days to $50 \%$ heading. The maximum values of such traits were obtained when barley plants received $60 \mathrm{~kg} \mathrm{~N} f e d^{-1}$ and foliar spray of $200 \mathrm{ppm}(\mathrm{Fe}+\mathrm{Zn}+\mathrm{Mn})$ for each element plus $1500 \mathrm{ppm}$ citric acid.
\end{abstract}

Key word: Barley, nitrogen, micronutrients, citric acid.

\section{INTRODUCTION}

In Egypt, barley (Hordem vulgare L.) is the main cereal crop grown in rainfed areas and new reclaimed lands. Barley is considered one of the most important cereal crops in the world being used for many purposes such as malting and brewing industry, animal feeding, bread making alone or by mixing with wheat flour in some places, some human food and beverages and many other uses. In Egypt, most of barley production areas are located where the adverse conditions exist such as poor soil. Nitrogen fertilization is a common agronomic practice that leads to improve barley productivity. In Egypt, new lands such as calcareous soils need more studies to determine macro and micro nutrient needs. Many researches have postulated the importance of nitrogen fertilization in improving growth, yield and yield components for barley crop. Dirienzo et al. (1991) reported that increasing nitrogen fertilizer rates i. e. 0,40 ,
80 and $120 \mathrm{~b} \mathrm{~N} \mathrm{acre}^{-1}$ increased grain and straw yields as well as crude protein of barley grains. Megahed et al. (1999) reported that, adding nitrogen fertilizer i.e. $0,107,143$ and $179 \mathrm{~kg} \mathrm{~N}$ $\mathrm{ha}^{-1}$ to barley plants (Giza 124), increased significantly plant height, biological yield, grain and straw yields by adding $179 \mathrm{~kg} \mathrm{~N} \mathrm{ha}^{-1}$ while, heading date was significantly decreased. Megahed et al. (2001 b) indicated that plant height, spike length, number of spike $/ \mathrm{m}^{-2}$, number of kernels /spike, spike kernel weight, 1000-kernel weight, biological yield, grain and straw yields of barley significantly increased with increasing nitrogen levels up to $90 \mathrm{~kg} \mathrm{fed}^{-1}$. Roy and Singh (2006) noted that adding nitrogen fertelizer up to $90 \mathrm{~kg} \mathrm{~N} \mathrm{ha}{ }^{-1}$ significantly increased grain and straw yields. Zeidan (2007) stated that increasing nitrogen rates from 30 to $70 \mathrm{~kg} \mathrm{~N} \mathrm{fed}{ }^{-1}$ increased plant height, flag leaf area, the number of spike $\mathrm{m}^{-2}, 1000$-kernels weight and grain yield as well as protein content 
of barley grains. Abd El-Rahman et al. (2012) stated that, the maximum values of plant height and spike length were obtained when barley received $45 \mathrm{~kg} \mathrm{~N} \mathrm{fed}^{-1}$. In newly reclaimed calcareous soils micronutrients play a great role to improve barley production. Abdel-Hadi et al. (1990) found that applying $\mathrm{Zn}$ either to the soil or as a foliar spray led to a marked increase for wheat grain yield. El-Sayed and Abdel-Hadi (1991) found a significant increase in plant height, grain yield and harvest index for barley grown in sandy soil by spraying plants with micronutrient a mixture of $\mathrm{Fe}+\mathrm{Zn}+\mathrm{Mn}$. ElHabbal et al. (1995), found that coating wheat grains with a mixture prepared from three individual chelating micronutrients each contained $12 \%$ of $\mathrm{Fe}, \mathrm{Mn}$ and $\mathrm{Zn}$ gave the highest grain and straw yields. Anton et al. (1999), reported that, coating barley grains with a micronutrient mixture $(\mathrm{Fe}+\mathrm{Zn}+\mathrm{Mn})$ significantly increased plant height, number of tillers $\mathrm{m}^{-2}$, the number of spikes $\mathrm{m}^{-2}$, spike length, spike kernels weight, 1000-kernel weight as well as straw, grain and biological yields fed $^{-}$ 1. Citric acid has a simulative effect on plant growth and development. Miernyk and Trelease (1981) found that citric acid is one of the organic acids presented in tricarboxylic acid cycle and synthesized either from acetyl-COA, glycine and $\alpha$-ketoglutic, or malic acid conversion to citric acid.

The objective of this study was to investigate the effect of nitrogen fertilization and foliar spray with a micronutrient mixture $(\mathrm{Fe}+\mathrm{Zn}+$ $\mathrm{Mn}$ ) and citric acid on barley growth, yield and yield components as well as the total chlorophyll of leaves and protein content of grains under calcareous soil conditions.

\section{MATERIALS AND METHODS}

The present work was carried out at Nubaria Agricultural Research Station, El-Behira Governorate, Egypt, during 2012/2013 and $2013 / 2014$ seasons. The aim was to study the effect of nitrogen fertilizer rates i. e. 15, 30, 45, and $60 \mathrm{~kg} \mathrm{~N} \mathrm{fed}{ }^{-1}$ and foliar spray with a micronutrients mixture of $\mathrm{Fe}+\mathrm{Zn}+\mathrm{Mn}$ at aconcentration of 0,133 and $200 \mathrm{ppm}$ for each element and citric acid $(0,1000$ and $1500 \mathrm{ppm})$ on barley Giza 2000 cultivar. Citrin solution consists of $2 \% \mathrm{Fe}, \mathrm{Zn}$ and $\mathrm{Mn}$ for each element plus $15 \%$ citric acid was used. Citrin solution was supplied by the biofertilizer production unit, soils, water and environment Research Institute, Agric. Res. Center (ARC), Giza, Egypt. The experiment was laid out in a split plot design with four replicates. The main plots were occupied by nitrogen levels and sub-plots contained micronutrients mixture plus citric acid. Sub-plot area was $10.5 \mathrm{~m}^{2}$ (3.5 x 3m), 15 rows, $20 \mathrm{~cm}$ apart and 3.5 long. Some physical and chemical properties of the experimental site shown in Table (1) and were done according to Ryan et al. (1996) The treatments were as follows:-

- Main plots (Nitrogen fertilization) were four rates of $\mathrm{N} \mathrm{15,30,45}$ and $60 \mathrm{~kg} \mathrm{fed}^{-1}$.

- Sub-plots (micronutrients mixture + citric acid):- spray with water $\left(S_{0}\right)$, spray with 133 $\mathrm{ppm}(\mathrm{Fe}+\mathrm{Zn}+\mathrm{Mn})$ for each elements plus $1000 \mathrm{ppm}$ citric acid $\left(\mathrm{S}_{1}\right)$ and spray with 200 $\mathrm{ppm}(\mathrm{Fe}+\mathrm{Zn}+\mathrm{Mn})$ for each with elements plus $1500 \mathrm{ppm}$ citric acid.

Table (1): Physical and chemical properties of the experimental site in both seasons.

\begin{tabular}{|c|c|c|}
\hline Property & $2012 / 2013$ & $2013 / 2014$ \\
\hline \multicolumn{3}{|l|}{ Particle size distribution } \\
\hline sand \% & 51.4 & 27.54 \\
\hline Silt \% & 24.9 & 36.76 \\
\hline Clay \% & 23.7 & 24.67 \\
\hline Texture & Sandy clay loam & Sandy clay loam \\
\hline PH & $7.5^{\circ}$ & 7.1 \\
\hline O. M\% (Organic matter \%) & 0.21 & 0.18 \\
\hline $\mathrm{CaCO}_{3}$ & 18.10 & 19.10 \\
\hline \multicolumn{3}{|l|}{ Available nutrients $\left(\mathrm{mg} \mathrm{kg}^{-1}\right)$} \\
\hline $\mathbf{N}$ & 12.50 & 13.20 \\
\hline $\mathbf{P}$ & 4.10 & 3.95 \\
\hline $\mathbf{K}$ & 115.00 & 126.00 \\
\hline \multicolumn{3}{|l|}{$\begin{array}{l}\text { DTPA-extractable }\left(\mathrm{mg} \mathrm{kg}^{-1}\right) \\
\text { DT }\end{array}$} \\
\hline $\mathbf{F e}$ & 0.68 & 0.61 \\
\hline $\mathbf{Z n}$ & 0.41 & 0.39 \\
\hline Mn & 0.54 & 0.48 \\
\hline
\end{tabular}

*Textural classes according to the triangular diagram. C.F. Soil and Water Research Institute, A. R. C., Egypt. 
Giza 2000 cultivar was sown on 25/11/2012 and 16/11/2013 in the first and second seasons, respectively. All sub-plots received the recommended dose of $\mathrm{P}$ and $\mathrm{K}$ fertilization. 30 $\mathrm{kg} \mathrm{p}_{2} \mathrm{O}_{5} \mathrm{fed}^{-1}$ in the form of calcium super phosphate $\left(15 \% \mathrm{p}_{2} \mathrm{O}_{5}\right)$ and $48 \mathrm{~kg} \mathrm{~K}_{2} \mathrm{O}$ fed $^{-1}$ in the form of potassium sulphate $\left(48 \% \mathrm{~K}_{2} \mathrm{O}\right)$ were added and incorporated into soil before sowing. Nitrogen fertilization treatments in the form of ammonium nitrate $(33.5 \% \mathrm{~N})$ were added in two equal doses, the first at 21 days after sowing (DAS) and the second at 35 DAS. Foliar application of the micronutrient mixture and citric acid were sprayed two times, the first after 35 days from sowing (tillering stage) and the second after 50 days from sowing (before the heading stage), the volume of water was one liter/ plot, $0.5 \%$ wetting agent of 20 was used. Cultural practices were practiced according to the methods being adopted for growing barley in the locality.

\section{I. Growth traits}

Plant height $(\mathrm{cm})$, spike length $(\mathrm{cm})$, the number of days to $50 \%$ heading, crop growth rate (CGR) g /plant / week, leaf area / main stem $\left(\mathrm{cm}^{2}\right)$ were estimated for five plants by using leaf area meter at $75 \mathrm{DAS}$. For determining the crop growth rate (CGR), five plants were randomly taken from the outer rows of each subplots for the four replications. The sampling dates were 60, 75 and 90 DAS. Plants were separated into roots and shoots and then dried at $70 \mathrm{c}^{\circ}$ in a ventilated oven to a constant weight. The following formula was used to determine CGR according to Watson (1952) :

$\mathrm{CGR}=\left(\mathrm{W}_{2}-\mathrm{W}_{1}\right) /\left(\mathrm{t}_{2}-\mathrm{t}_{1}\right)$

Where: $\mathrm{W}_{2}-\mathrm{W}_{1}=$ differences in dry mater accumulation between two successive samples in grams. $t_{2}-t_{1}=$ the number of days between two successive samples in week.

Leaf area/main stem in $\mathrm{cm}^{2}$ was estimated for plants by using leaf area meter at 75 DAS.

\section{2. Yield and its components}

Harvest took place at 20/5/2013 and $10 / 5 / 2014$ in the first and second seasons, respectively. At harvest time, ten guarded plants were randomly taken from the central row in each sub-plot to determine the number of kernels / spike and 1000-kernel weight $(\mathrm{g})$. Number of spikes $/ \mathrm{m}^{2}$ was determined from one $\mathrm{m}^{2}$ area in each sub-plot. In addition, plants in the central area $\left(4 \mathrm{~m}^{2}\right)$ of each sub-plot were harvested to determine straw yield $\left(\mathrm{t} \mathrm{fed}^{-1}\right)$ and grain yield $\left(\operatorname{ard~fed}{ }^{-1}\right)$.

\section{3. Chemical traits}

Total chlorophyll content of the leaves at 75 DAS was determined as a SPAD unit (Soil and Plant Analysis Department) of Minolta Co. This unit was transformed to $\mathrm{mg} \mathrm{m}^{-2}$ as described by Monge and Bugbe (1992) as follows:- chl. = $80.05+10.4$ (SPAD 502). Grain samples from the two growing seasons were subjected to chemical analysis to determine crude protein\% in grains according to A. O. A. C. (1990).

The comparison of error mean squares between the two seasons for all traits was done by the help of Bartlett's test of homogeneity of variances (Snedecor and cochran, 1989) and found not significantly different.

Data of the two seasons were combined and statistically analyzed according to Steel and Torrie (1990). The discussion of the obtained results was carried out on the basis of the combined analysis values.

\section{RESULTS AND DISCUSSION 3. 1. Growth traits}

Results in Tables (2, 3 and 4) indicated that both nitrogen fertilization and foliar spray with micronutrients mixture plus citric acid had significant effects on plant height, leaf area / main stem, number of days to $50 \%$ heading, spike length as well as crop growth rate (CGR) at the first period (60-75 days) and the second one (75-90 days). It can be noticed that CGR values were higher in the first period than in the second period. Such decrease at the second period is mainly due to the fact that barley plants directed its effort to heading and grain formation. Results showed that increasing nitrogen fertilizer from $15 \mathrm{~kg} \mathrm{~N} \mathrm{fed}^{-1}$ up to $60 \mathrm{~kg}$ $\mathrm{N}$ fed ${ }^{-1}$ increased gradually all growth traits under study. Abdo and El-Moselhy. (2004) explained such finding to be the function of nitrogen in plant metabolism i. e. as constituent of amino and nucleic acids, many cofactors and cellular compounds. It is worthy to mention that insignificant differences were observed between adding $45 \mathrm{~kg} \mathrm{~N}$ fed $^{-1}$ and $60 \mathrm{~kg} \mathrm{~N}$ fed $^{-1}$ for all growth traits under study, except the number of days to $50 \%$ heading. These results are in harmony with those obtained by Megahed (2003).

Concerning foliar spray of micronutrients mixture plus citric acid, data in Tables (2, 3 and 4) indicated that spraying plants by $200 \mathrm{ppm}$ of $(\mathrm{Fe}+\mathrm{Zn}+\mathrm{Mn})$ for each element plus $1500 \mathrm{ppm}$ citric acid $\left(\mathrm{S}_{2}\right)$ significantly increased plant 
Table (2): Plant height (cm) and leaf area/main stem $\left(\mathrm{cm}^{2}\right)$ of barley as affected by nitrogen fertilization and foliar spray by micronutrients mixture plus citric acid in 2012/2013, 2013/2014 and combined two seasons.

\begin{tabular}{|c|c|c|c|c|c|c|c|c|}
\hline \multicolumn{3}{|c|}{ Treatments } & \multicolumn{3}{|c|}{ Plant height } & \multicolumn{3}{|c|}{$\begin{array}{c}\text { Leaf area/main stem }\left(\mathrm{cm}^{2}\right) \\
\text { at } 75 \text { DAS }\end{array}$} \\
\hline $\begin{array}{c}\text { Nitrogen } \\
\text { level } \mathrm{kg} \mathrm{N} \text { fed }^{-1}\end{array}$ & $\begin{array}{l}\text { Micr } \\
\text { mixtu } \\
\text { ac }\end{array}$ & $\begin{array}{l}\text { ients } \\
\text { citric } \\
\text { ) }\end{array}$ & $\begin{array}{l}2012 / \\
2013\end{array}$ & $\begin{array}{r}2013 / \\
2014\end{array}$ & Comb. & $\begin{array}{l}2012 / \\
2013\end{array}$ & $\begin{array}{r}2013 / \\
2014\end{array}$ & Comb. \\
\hline \multirow{3}{*}{15} & & & 61.25 & 65.50 & 63.38 & 96.82 & 97.19 & 97.01 \\
\hline & & & 67.5 & 67.50 & 67.50 & 111.84 & 109.49 & 110.67 \\
\hline & & & 72.25 & 72.50 & 72.38 & 116.82 & 114.34 & 115.58 \\
\hline \multicolumn{3}{|l|}{ Mean } & 67.00 & 68.50 & 67.75 & 108.49 & 107.01 & 107.75 \\
\hline \multirow{3}{*}{30} & & & 67.75 & 68.75 & 68.25 & 112.31 & 109.20 & 110.76 \\
\hline & & & 71.00 & 73.75 & 72.38 & 115.70 & 115.19 & 115.45 \\
\hline & & & 70.25 & 75.75 & 73.00 & 129.65 & 127.54 & 128.60 \\
\hline \multicolumn{3}{|l|}{ Mean } & 69.67 & 72.75 & 71.21 & 119 & 117.31 & 118.27 \\
\hline \multirow{3}{*}{45} & & & 76.50 & 79.00 & 77.75 & 126.39 & 124.59 & 125.49 \\
\hline & & & 82.00 & 83.00 & 82.50 & 134.31 & 132.46 & 133.39 \\
\hline & & & 85.75 & 88.25 & 87.00 & 141.01 & 139.23 & 140.12 \\
\hline \multicolumn{3}{|l|}{ Mean } & 81.42 & 83.42 & 82.42 & 133.90 & 132.09 & 133.00 \\
\hline \multirow{3}{*}{60} & & & 77.75 & 73.75 & 75.75 & 127.58 & 125.31 & 126.45 \\
\hline & & & 83.25 & 87.75 & 85.50 & 135.07 & 134.28 & 134.68 \\
\hline & & & 85.75 & 89.25 & 87.50 & 146.23 & 143.43 & 144.83 \\
\hline \multicolumn{3}{|l|}{ Mean } & 82.25 & 83.58 & 82.92 & 136.29 & 134.34 & 135.32 \\
\hline \multirow{3}{*}{\multicolumn{2}{|c|}{$\begin{array}{l}\text { General mean of } \\
\text { micronutrients + citric } \\
\text { acid }\end{array}$}} & $\mathbf{S}_{\mathbf{0}}$ & 70.81 & 71.75 & 71.28 & 115.78 & 114.07 & 114.93 \\
\hline & & $S_{1}$ & 75.94 & 78.00 & 76.97 & 124.23 & 122.86 & 123.55 \\
\hline & & $\mathbf{S}_{2}$ & 78.50 & 81.44 & 79.97 & 133.43 & 131.14 & 132.28 \\
\hline \multirow{3}{*}{ L. S. D at 0.05} & & $\mathbf{N}$ & 1.82 & 1.73 & 1.15 & 10.45 & 10.70 & 6.87 \\
\hline & & $\mathbf{S}$ & 1.43 & 1.24 & 0.87 & 8.33 & 8.66 & 5.52 \\
\hline & & $\mathbf{N} \times \mathbf{S}$ & 2.86 & 2.49 & 1.74 & 20.72 & 22.72 & 14.12 \\
\hline \multicolumn{3}{|l|}{ C. V \% } & 2.63 & 2.22 & 2.43 & 7.91 & 8.10 & 8.00 \\
\hline
\end{tabular}

* $\mathrm{S}_{0}=$ Spraying with water, $\mathrm{S}_{1=}$ Spraying with $133 \mathrm{ppm}(\mathrm{Fe}+\mathrm{Zn}+\mathrm{Mn})$ for each element $+1000 \mathrm{ppm}$ citric acid and $S_{2}=$ Spraying with $200 \mathrm{ppm}(\mathrm{Fe}+\mathrm{Zn}+\mathrm{Mn})$ for each element $+1500 \mathrm{ppm}$ citric acid.

height, leaf area / main stem, number of days to $50 \%$ heading, spike length as well as CGR at citric acid $\left(\mathrm{S}_{2}\right)$ significantly increased plant height, leaf area / main stem, number of days to $50 \%$ heading, spike length as well as CGR at (60-75 days) and (75-90 days) periods. These results could be explained on the basis that micronutrients must be presented during vegetative stage to get the normal growth. Where, manganese regulates the oxidation reduction system of iron. Also, zinc plays an important role in tryptophan synthesis which is a precursor of IAA. In addition it was reported that the ratio between micronutrients in plant tissues was very important for the accumulation of many nutrients in plants (Osawa, 1973). also, Mourad (2006) reported that plant height of grain sorghum reached its maximum value when plants sprayed with $500 \mathrm{ppm}$ of citric acid.
Regarding the interaction between nitrogen fertilization and foliar spray of micronutrients mixture plus citric acid, data in Tables (2, 3 and 4) show a significant effect on all growth traits except the number of days to $50 \%$ heading. The maximum values of plant height, leaf area/main stem, spike length as well as crop growth rate at (60-75 days) and (75-90 days) periods were obtained when barley plants received $60 \mathrm{~kg} \mathrm{~N}$ fed $^{-1}$ and foliar application of $200 \mathrm{ppm}(\mathrm{Fe}+\mathrm{Zn}$ $+\mathrm{Mn}$ ) for each element plus $1500 \mathrm{ppm}$ of citric acid.

\section{2. Yield and its components traits}

Data in Tables (5, 6 and 7) showed that nitrogen fertilization had a significant effect on the number of spikes $/ \mathrm{m}^{2}$, number of kernels / spike, 1000-kernel weight as well as straw and grain yields. The highest values of such traits were scored from applying $60 \mathrm{~kg} \mathrm{~N}^{-1} \mathrm{fed}^{-1}$. This 
result could be attributed to the role of nitrogen on barley growth and yield components which in turn reflected on straw and grain yields/fed. These results are supported by those obtained by Roy and Singh (2006), Zeidan (2007) and Abd El-Rahman et al.(2012). It is worthy to mention that, insignificant differences were observed between adding 45 and $60 \mathrm{~kg} \mathrm{~N}$ fed $^{-1}$ with respect to grain yield of barley. So, from the economic point of view and nitrogen fertilizer conservation, it is advisable to practice $45 \mathrm{~kg} \mathrm{~N}$ fed $^{-1}$ under calcareous soils condition in Nubaria region.

As for the effect of foliar spray of micronutrient mixture plus citric acid (Tables 5, 6 and 7), the results indicated that, spraying $200 \mathrm{ppm}(\mathrm{Fe}+\mathrm{Zn}+\mathrm{Mn})$ for each element plus $1500 \mathrm{ppm}$ citric acid $\left(\mathrm{S}_{2}\right)$ on barley plants significantly increased number of spikes $\mathrm{m}^{-2}$, the number of kernels spike ${ }^{-1}$, 1000-kernel weight (g), straw yield $\left(\mathrm{t} \mathrm{fed}^{-1}\right)$ and grain yield $\left(\operatorname{ard~fed}^{-1}\right)$ compared with other foliar spray treatments $\left(S_{0}\right.$ and $S_{1}$ ). In this connection, Anton et al. (1999) found that foliar spray of ascorbic and citric acids in combination with micronutrients mixture $(\mathrm{Fe}+\mathrm{Zn}+\mathrm{Mn})$ on barley plants had a significant effect on plant height, the number of tillers $\mathrm{m}^{-2}$, straw and biological yields $\mathrm{fed}^{-1}$.

Concerning the interaction effect between nitrogen fertilization and foliar spray of micronutrients mixture plus citric acid, data in Tables (5, 6 and 7) showed that all yield and yield components traits under study recorded a significant effect. The maximum values of number of spikes $\mathrm{m}^{-2}$, number of kernels spike${ }^{1}, 1000$-kernel weight $(\mathrm{g})$, straw yield $\left(\mathrm{t} \mathrm{fed}^{-1}\right)$ and grain yield $\left(\right.$ ard $\left.\mathrm{fed}^{-1}\right)$ were obtained when barley plants were treated by $60 \mathrm{~kg} \mathrm{~N}^{-1}$ and foliar spray with of $200 \mathrm{ppm}(\mathrm{Fe}+\mathrm{Zn}+\mathrm{Mn})$ for each element plus $1500 \mathrm{ppm}$ citric acid $\left(\mathrm{S}_{2}\right)$.

Table (3): Number of days to 50\% heading and spike length (cm) of barley as affected by nitrogen fertilization and foliar spray by micronutrients mixture plus citric acid in 2012/2013, 2013/2014 and combined two seasons.

\begin{tabular}{|c|c|c|c|c|c|c|c|c|}
\hline \multicolumn{3}{|c|}{ Treatments } & \multicolumn{3}{|c|}{$\begin{array}{c}\text { Number of days to } 50 \% \\
\text { heading }\end{array}$} & \multicolumn{3}{|c|}{ Spike length (cm) } \\
\hline $\begin{array}{c}\text { Nitrogen } \\
\text { level kg } \mathrm{fed}^{-1}\end{array}$ & $\begin{array}{l}\text { Mi } \\
\text { mix }\end{array}$ & $\begin{array}{l}\text { utrients } \\
+ \text { citric } \\
*(\mathbf{S})\end{array}$ & $\begin{array}{c}2012 / \\
2013\end{array}$ & $\begin{array}{l}2013 / \\
2014\end{array}$ & Comb. & $\begin{array}{l}2012 / \\
2013\end{array}$ & $\begin{array}{l}2013 / \\
2014\end{array}$ & Comb. \\
\hline \multirow{3}{*}{15} & & $0_{0}$ & 83.25 & 82.75 & 83.00 & 5.22 & 5.33 & 5.28 \\
\hline & & $y_{1}$ & 84.25 & 83.50 & 83.88 & 5.73 & 5.17 & 5.45 \\
\hline & & $2_{2}$ & 84.75 & 85.25 & 85.00 & 5.96 & 6.19 & 6.08 \\
\hline \multicolumn{3}{|c|}{ Mean } & 84.08 & 83.83 & 83.96 & 5.64 & 5.56 & 5.60 \\
\hline \multirow{3}{*}{30} & & $s_{0}$ & 85.00 & 84.75 & 84.88 & 6.23 & 6.26 & 6.25 \\
\hline & & $S_{1}$ & 86.00 & 85.50 & 85.75 & 6.61 & 6.60 & 6.61 \\
\hline & & $y_{2}$ & 86.25 & 86.50 & 86.38 & 6.81 & 6.92 & 6.87 \\
\hline \multicolumn{3}{|c|}{ Mean } & 85.75 & 85.58 & 85.67 & 6.55 & 6.59 & 6.57 \\
\hline \multirow{3}{*}{45} & & $s_{0}$ & 86.75 & 86.75 & 86.75 & 7.24 & 7.22 & 7.23 \\
\hline & & $y_{1}$ & 87.75 & 87.25 & 87.50 & 7.57 & 7.60 & 7.59 \\
\hline & & 2 & 88.25 & 87.75 & 88.00 & 7.83 & 7.58 & 7.71 \\
\hline \multicolumn{3}{|c|}{ Mean } & 87.58 & 87.25 & 87.42 & 7.55 & 7.47 & 7.51 \\
\hline \multirow{3}{*}{60} & & o & 88.50 & 88.50 & 88.50 & 6.68 & 6.99 & 6.84 \\
\hline & & 8 & 88.75 & 88.50 & 88.63 & 7.77 & 7.90 & 7.84 \\
\hline & & $y_{2}$ & 89.00 & 88.75 & 88.88 & 8.34 & 8.06 & 8.20 \\
\hline \multicolumn{3}{|c|}{ Mean } & 88.75 & 88.58 & 88.67 & 7.60 & 7.65 & 7.63 \\
\hline \multirow{3}{*}{\multicolumn{2}{|c|}{$\begin{array}{l}\text { General mean of } \\
\text { micronutrients + citric } \\
\text { acid }\end{array}$}} & $\mathbf{S}_{\mathbf{0}}$ & 85.88 & 85.69 & 85.78 & 6.34 & 6.45 & 6.39 \\
\hline & & $\mathbf{S}_{1}$ & 86.69 & 86.19 & 86.72 & 6.92 & 6.95 & 6.94 \\
\hline & & $S_{2}$ & 87.06 & 87.06 & 87.06 & 7.23 & 7.19 & 7.21 \\
\hline \multirow{3}{*}{\multicolumn{2}{|c|}{ L. S. D at 0.05}} & $\mathbf{N}$ & 0.84 & 0.80 & 0.53 & 0.23 & 0.22 & 0.15 \\
\hline & & $\mathbf{S}$ & 0.65 & 0.56 & 0.39 & 0.20 & 0.17 & 0.12 \\
\hline & & $\mathrm{N} \times \mathrm{S}$ & N. S & N. S & N. S & 0.36 & 0.31 & 0.22 \\
\hline \multicolumn{3}{|l|}{ C. V \% } & 0.75 & 0.90 & 0.82 & 2.05 & 4.03 & 3.20 \\
\hline
\end{tabular}

$* \mathrm{~S}_{0}=$ Spraying with water, $\mathrm{S}_{1=}$ Spraying with $133 \mathrm{ppm}(\mathrm{Fe}+\mathrm{Zn}+\mathrm{Mn})$ for each element $+1000 \mathrm{ppm}$ citric acid and $\mathrm{S}_{2}=$ Spraying with $200 \mathrm{ppm}(\mathrm{Fe}+\mathrm{Zn}+\mathrm{Mn})$ for each element $+1500 \mathrm{ppm}$ citric acid. 
Table (4): Crop growth rate (CGR) g/plant/week at (60-75) and (75-90) DAS of barley as affected by nitrogen fertilization and foliar spray by micronutrients mixture plus citric acid in 2012/2013, 2013/2014 and combined two seasons.

\begin{tabular}{|c|c|c|c|c|c|c|c|c|}
\hline \multirow{2}{*}{\multicolumn{3}{|c|}{ Treatments }} & \multicolumn{6}{|c|}{ Crop growth rate (CGR) g/plant/week } \\
\hline & & & & 0-75) DAS & & & 5-90) DAS & \\
\hline $\begin{array}{c}\text { Nitrogen } \\
\text { level } \mathrm{kg} \mathrm{N} \text { fed }^{-1}\end{array}$ & \multicolumn{2}{|c|}{$\begin{array}{c}\text { Micronutrients mixture + } \\
\text { citric acid } *(\mathbf{S})\end{array}$} & $2012 / 2013$ & $2013 / 2014$ & Comb & $2012 / 2013$ & $2013 / 2014$ & Comb \\
\hline \multirow{3}{*}{15} & \multicolumn{2}{|c|}{$\mathbf{S}_{\mathbf{0}}$} & 0.97 & 0.71 & 0.84 & 0.49 & 0.46 & 0.48 \\
\hline & \multicolumn{2}{|c|}{$S_{1}$} & 1.08 & 0.95 & 1.02 & 0.56 & 0.53 & 0.55 \\
\hline & \multicolumn{2}{|c|}{$S_{2}$} & 1.10 & 1.05 & 1.08 & 0.59 & 0.58 & 0.57 \\
\hline \multicolumn{3}{|c|}{ Mean } & 1.05 & 0.90 & 0.98 & 0.54 & 0.52 & 0.53 \\
\hline \multirow{3}{*}{30} & \multicolumn{2}{|c|}{$S_{0}$} & 1.04 & 0.84 & 0.94 & 0.54 & 0.53 & 0.54 \\
\hline & \multicolumn{2}{|c|}{$S_{1}$} & 1.15 & 1.03 & 1.09 & 0.62 & 0.61 & 0.62 \\
\hline & \multicolumn{2}{|c|}{$S_{2}$} & 1.17 & 1.10 & 1.14 & 0.64 & 0.62 & 0.63 \\
\hline \multicolumn{3}{|c|}{ Mean } & 1.12 & 0.99 & 1.06 & 0.60 & 0.59 & 0.60 \\
\hline \multirow{3}{*}{45} & \multicolumn{2}{|c|}{$\mathbf{S}_{\mathbf{0}}$} & 1.09 & 1.02 & 1.06 & 0.60 & 0.59 & 0.60 \\
\hline & \multicolumn{2}{|c|}{$S_{1}$} & 1.20 & 1.12 & 1.16 & 0.70 & 0.64 & 0.67 \\
\hline & \multicolumn{2}{|c|}{$S_{2}$} & 1.23 & 1.13 & 1.18 & 0.71 & 0.68 & 0.70 \\
\hline \multicolumn{3}{|c|}{ Mean } & 1.17 & 1.09 & 1.13 & 0.67 & 0.64 & 0.66 \\
\hline \multirow{3}{*}{60} & \multicolumn{2}{|c|}{$S_{0}$} & 1.18 & 1.09 & 1.14 & 0.63 & 0.60 & 0.62 \\
\hline & \multicolumn{2}{|c|}{$S_{1}$} & 1.22 & 1.13 & 1.18 & 0.71 & 0.70 & 0.71 \\
\hline & \multicolumn{2}{|c|}{$\mathbf{S}_{2}$} & 1.24 & 1.14 & 1.19 & 0.72 & 0.74 & 0.73 \\
\hline \multicolumn{3}{|c|}{ Mean } & 1.21 & 1.12 & 1.17 & 0.70 & 0.68 & 0.69 \\
\hline \multirow{3}{*}{\multicolumn{2}{|c|}{$\begin{array}{l}\text { General mean of } \\
\text { micronutrients + citric acid }\end{array}$}} & $\mathbf{S}_{\mathbf{0}}$ & 1.07 & 0.92 & 0.99 & 0.57 & 0.55 & 0.56 \\
\hline & & $S_{1}$ & 1.16 & 1.06 & 1.11 & 0.65 & 0.62 & 0.64 \\
\hline & & $\mathbf{S}_{2}$ & 1.19 & 1.11 & 1.15 & 0.67 & 0.66 & 0.66 \\
\hline \multirow{3}{*}{\multicolumn{2}{|c|}{ L. S. D at 0.05}} & $\mathbf{N}$ & 0.10 & 0.11 & 0.07 & 0.08 & 0.09 & 0.06 \\
\hline & & $\mathbf{S}$ & 0.07 & 0.08 & 0.05 & 0.06 & 0.07 & 0.04 \\
\hline & & $\mathbf{N} \times \mathbf{S}$ & 0.17 & 0.20 & 0.12 & 0.15 & 0.18 & 0.11 \\
\hline \multicolumn{3}{|l|}{ C. V\% } & 8.64 & 8.91 & 8.78 & 9.31 & 8.15 & 0.73 \\
\hline
\end{tabular}

${ }^{*} \mathrm{~S}_{0}=$ Spraying with water, $\mathrm{S}_{1=}$ Spraying with $133 \mathrm{ppm}(\mathrm{Fe}+\mathrm{Zn}+\mathrm{Mn})$ for each element $+1000 \mathrm{ppm}$ citric acid and

$\mathrm{S}_{2}=$ Spraying with $200 \mathrm{ppm}(\mathrm{Fe}+\mathrm{Zn}+\mathrm{Mn})$ for each element $+1500 \mathrm{ppm}$ citric acid

Table (5): Number of spikes $/ \mathrm{m}^{2}$ and number of kernels / spike of barley as affected by nitrogen fertilization and foliar spray by micronutrients mixture plus citric acid in 2012/2013, 2013/2014 and combined two seasons.

\begin{tabular}{|c|c|c|c|c|c|c|c|c|}
\hline \multicolumn{3}{|c|}{ Treatments } & \multicolumn{3}{|c|}{ Number of spikes $/ \mathrm{m}^{2}$} & \multicolumn{3}{|c|}{ Number of kernels / spike } \\
\hline $\begin{array}{c}\text { Nitrogen } \\
\text { level } \mathrm{kg}_{\mathrm{N}} \text { fed }^{-1}\end{array}$ & $\begin{array}{r}\text { Micronu } \\
\text { citr }\end{array}$ & $\begin{array}{l}\text { ents mixture + } \\
\text { acid } *(\mathbf{S})\end{array}$ & $2012 / 2013$ & $2013 / 2014$ & Comb. & $2012 / 2013$ & $2013 / 2014$ & Comb. \\
\hline \multirow{3}{*}{15} & & $\mathbf{S}_{\mathbf{0}}$ & 339.75 & 331.25 & 335.50 & 31.50 & 31.75 & 31.63 \\
\hline & & $S_{1}$ & 350.00 & 338.00 & 344.00 & 34.50 & 34.00 & 34.25 \\
\hline & & $S_{2}$ & 355.75 & 345.00 & 350.38 & 35.50 & 33.25 & 35.38 \\
\hline \multicolumn{3}{|c|}{ Mean } & 348.50 & 338.08 & 343.30 & 33.83 & 33.75 & 33.75 \\
\hline \multirow{3}{*}{30} & & $\mathbf{S}_{\mathbf{0}}$ & 365.75 & 353.25 & 359.50 & 38.50 & 37.50 & 38.00 \\
\hline & & $\mathbf{S}_{1}$ & 373.25 & 359.75 & 366.50 & 40.50 & 40.75 & 40.63 \\
\hline & & $\mathbf{S}_{2}$ & 375.50 & 365.75 & 370.38 & 41.75 & 41.75 & 41.75 \\
\hline \multicolumn{3}{|c|}{ Mean } & 371.50 & 359.42 & 365.45 & 40.25 & 40.00 & 40.13 \\
\hline \multirow{3}{*}{45} & & $\mathbf{S}_{\mathbf{0}}$ & 382.50 & 373.75 & 378.13 & 42.95 & 43.50 & 42.98 \\
\hline & & $S_{1}$ & 386.00 & 373.85 & 379.87 & 45.91 & 46.50 & 46.08 \\
\hline & & $\mathbf{S}_{2}$ & 391.00 & 376.25 & 383.63 & 48.50 & 48.75 & 48.63 \\
\hline \multicolumn{3}{|c|}{ Mean } & 386.50 & 374.58 & 380.54 & 45.79 & 46.00 & 45.89 \\
\hline \multirow{3}{*}{60} & & $S_{0}$ & 375.00 & 381.00 & 378.00 & 42.50 & 43.00 & 42.75 \\
\hline & & $S_{1}$ & 386.00 & 389.25 & 387.62 & 44.50 & 45.75 & 45.13 \\
\hline & & $S_{2}$ & 393.00 & 393.75 & 393.37 & 51.75 & 52.75 & 52.25 \\
\hline \multicolumn{3}{|c|}{ Mean } & 384.67 & 388.00 & 386.33 & 46.25 & 47.63 & 46.71 \\
\hline \multirow{3}{*}{\multicolumn{2}{|c|}{$\begin{array}{l}\text { General mean of } \\
\text { micronutrients + citric acid }\end{array}$}} & $\mathbf{S}_{\mathbf{0}}$ & 365.75 & 359.81 & 362.78 & 38.86 & 38.81 & 38.84 \\
\hline & & $\mathbf{S}_{1}$ & 373.81 & 365.19 & 369.50 & 41.35 & 41.69 & 41.52 \\
\hline & & $S_{2}$ & 378.81 & 370.06 & 374.43 & 44.38 & 44.63 & 44.50 \\
\hline \multirow{3}{*}{\multicolumn{2}{|c|}{ L. S. D at 0.05}} & $\mathbf{N}$ & 6.58 & 6.25 & 4.17 & 0.76 & 0.88 & 0.53 \\
\hline & & $\mathbf{S}$ & 5.17 & 4.91 & 3.28 & 0.63 & 0.66 & 0.42 \\
\hline & & $\mathbf{N} \times \mathbf{S}$ & 10.34 & 9.82 & 6.55 & 1.27 & 1.32 & 0.90 \\
\hline \multicolumn{3}{|l|}{ C. V \% } & 11.06 & 11.34 & 11.06 & 2.11 & 2.19 & 2.15 \\
\hline
\end{tabular}

$* \mathrm{~S}_{0}=$ Spraying with water, $\mathrm{S}_{1=}$ Spraying with $133 \mathrm{ppm}(\mathrm{Fe}+\mathrm{Zn}+\mathrm{Mn})$ for each element $+1000 \mathrm{ppm}$ citric acid and $\mathrm{S}_{2}=$ Spraying with $200 \mathrm{ppm}$ $(\mathrm{Fe}+\mathrm{Zn}+\mathrm{Mn})$ for each element $+1500 \mathrm{ppm}$ citric acid. 
Table (6): 1000-kernel weight (g) and Straw yield (T/fed) of barley as affected by nitrogen fertilization and foliar spray by micronutrients mixture plus citric acid in 2012/2013, 2013/2014 and combined two seasons.

\begin{tabular}{|c|c|c|c|c|c|c|c|c|}
\hline \multicolumn{3}{|c|}{ Treatments } & \multicolumn{3}{|c|}{ 1000-kernel weight $(\mathrm{g})$} & \multicolumn{3}{|c|}{ Straw yield (T/fad) } \\
\hline 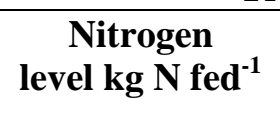 & $\begin{array}{r}\text { Micro } \\
\text { mixture }\end{array}$ & $\begin{array}{l}\text { ents } \\
\text { ic acid }\end{array}$ & $\begin{array}{l}2012 / \\
2013\end{array}$ & $\begin{array}{l}2013 / \\
2014\end{array}$ & Comb. & $2012 /$ & $2013 /$ & Comb. \\
\hline \multirow{3}{*}{15} & \multicolumn{2}{|c|}{$\mathbf{S}_{0}$} & 31.45 & 31.49 & 31.45 & 3.22 & 3.22 & 3.21 \\
\hline & \multicolumn{2}{|c|}{$\mathbf{S}_{1}$} & 34.45 & 34.55 & 34.50 & 3.34 & 3.35 & 3.34 \\
\hline & \multicolumn{2}{|c|}{$\mathbf{S}_{2}$} & 35.43 & 35.60 & 35.51 & 3.43 & 3.45 & 3.44 \\
\hline \multicolumn{3}{|c|}{ Mean } & 33.78 & 33.85 & 33.82 & $\mathbf{3 . 3 3}$ & 3.34 & 3.33 \\
\hline \multicolumn{3}{|c|}{$\overline{S_{0}}$} & 38.55 & 38.43 & 38.55 & 3.34 & 3.37 & 3.36 \\
\hline \multirow[t]{2}{*}{30} & \multicolumn{2}{|c|}{$\mathbf{S}_{1}$} & 39.85 & 39.85 & 39.85 & 3.53 & 3.56 & 3.55 \\
\hline & \multicolumn{2}{|c|}{$\mathbf{S}_{2}$} & 40.53 & 40.58 & 40.53 & 3.67 & 3.71 & 3.69 \\
\hline \multicolumn{3}{|c|}{ Mean } & 39.64 & 39.64 & 39.64 & 3.51 & $\mathbf{3 . 5 5}$ & 3.53 \\
\hline \multicolumn{3}{|c|}{$\mathbf{S}_{\mathbf{0}}$} & 42.53 & 42.63 & 42.53 & 3.43 & 3.54 & 3.49 \\
\hline \multirow[t]{2}{*}{45} & \multicolumn{2}{|c|}{$\mathbf{S}_{1}$} & 45.05 & 45.13 & 45.05 & 3.77 & 3.81 & 3.79 \\
\hline & \multicolumn{2}{|c|}{$\mathbf{S}_{2}$} & 46.58 & 46.61 & 46.58 & 4.13 & 4.04 & 4.08 \\
\hline \multicolumn{3}{|c|}{ Mean } & 44.72 & 44.70 & 44.76 & 3.78 & 3.80 & 3.79 \\
\hline \multicolumn{3}{|c|}{$\mathbf{S}_{\mathbf{0}}$} & 48.05 & 48.13 & 48.08 & 3.62 & 3.51 & 3.57 \\
\hline \multirow[t]{2}{*}{60} & \multicolumn{2}{|c|}{$\mathbf{S}_{1}$} & 48.95 & 48.83 & 48.95 & 3.16 & 4.17 & 4.17 \\
\hline & \multicolumn{2}{|c|}{$\mathbf{S}_{2}$} & 49.63 & 49.63 & 49.63 & 4.44 & 4.40 & 4.42 \\
\hline \multicolumn{3}{|c|}{ Mean } & 48.87 & 48.88 & 48.51 & 4.08 & 4.02 & 4.05 \\
\hline \multirow{3}{*}{\multicolumn{2}{|c|}{$\begin{array}{l}\text { General mean of } \\
\text { micronutrients + citric acid }\end{array}$}} & $\mathbf{S}_{\mathbf{0}}$ & 40.14 & 40.14 & 40.14 & 3.40 & 3.41 & 3.41 \\
\hline & & $\mathbf{S}_{1}$ & 42.07 & 42.10 & 42.09 & 3.70 & 3.72 & 3.71 \\
\hline & & $\mathbf{S}_{2}$ & 43.04 & 43.08 & 43.08 & 3.92 & 3.90 & 3.90 \\
\hline \multirow[t]{3}{*}{ L. S. D at 0.05} & & $\mathbf{N}$ & 0.93 & 0.98 & 0.67 & 0.18 & 0.17 & 0.11 \\
\hline & & $\mathbf{S}$ & 0.76 & 0.66 & 0.46 & 0.14 & 0.13 & 0.09 \\
\hline & & $\mathbf{N} \times S$ & 1.19 & 1.32 & 0.82 & 0.20 & 0.22 & 0.16 \\
\hline \multicolumn{3}{|l|}{ C. V\% } & 2.85 & 2.87 & 2.86 & 11.30 & 12.35 & 11.90 \\
\hline
\end{tabular}

$* \mathrm{~S}_{0}=$ Spraying with water, $\mathrm{S}_{1=}$ Spraying with $133 \mathrm{ppm}(\mathrm{Fe}+\mathrm{Zn}+\mathrm{Mn})$ for each element $+1000 \mathrm{ppm}$ citric acid and $S_{2}=$ Spraying with $200 \mathrm{ppm}(\mathrm{Fe}+\mathrm{Zn}+\mathrm{Mn})$ for each element $+1500 \mathrm{ppm}$ citric acid. 


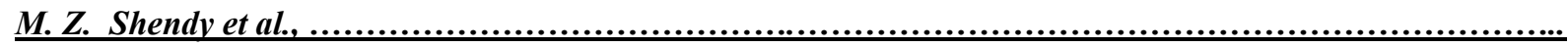

Table (7): Grain yield (ard/fed) of barley as affected by nitrogen fertilization and foliar spray by micronutrients mixture plus citric acid in 2012/2013, 2013/2014 and combined two seasons.

\begin{tabular}{|c|c|c|c|c|c|}
\hline \multirow{2}{*}{$\begin{array}{c}\text { Nitrogen } \\
\text { level } \mathrm{kg} \mathrm{N} \mathrm{fed}^{-1}\end{array}$} & \multirow{2}{*}{\multicolumn{2}{|c|}{$\begin{array}{l}\text { Micronutrients mixture + } \\
\quad \text { citric acid } *(S)\end{array}$}} & \multicolumn{3}{|c|}{ Grain yield (ard/fed) } \\
\hline & & & $2012 / 2013$ & $2013 / 2014$ & Comb. \\
\hline \multirow{3}{*}{15} & \multicolumn{2}{|c|}{$\mathbf{S}_{\mathbf{0}}$} & 10.56 & 10.61 & 10.59 \\
\hline & \multicolumn{2}{|c|}{$\mathbf{S}_{1}$} & 10.69 & 10.71 & 10.70 \\
\hline & \multicolumn{2}{|c|}{$\mathbf{S}_{2}$} & 10.84 & 10.81 & 10.83 \\
\hline \multicolumn{3}{|c|}{ Mean } & 10.70 & 10.71 & 10.71 \\
\hline \multirow{3}{*}{30} & \multicolumn{2}{|c|}{$\mathbf{S}_{\mathbf{0}}$} & 11.27 & 11.43 & 11.35 \\
\hline & \multicolumn{2}{|c|}{$S_{1}$} & 11.56 & 11.71 & 11.64 \\
\hline & \multicolumn{2}{|c|}{$\mathbf{S}_{\mathbf{2}}$} & 11.77 & 11.92 & 11.85 \\
\hline \multicolumn{3}{|c|}{ Mean } & 11.53 & 11.69 & 11.61 \\
\hline \multirow{3}{*}{45} & \multicolumn{2}{|c|}{$\mathbf{S}_{\mathbf{0}}$} & 12.56 & 13.21 & 12.89 \\
\hline & \multicolumn{2}{|c|}{$\mathbf{S}_{1}$} & 13.70 & 13.78 & 13.74 \\
\hline & \multicolumn{2}{|c|}{$\mathbf{S}_{2}$} & 14.65 & 14.87 & 14.76 \\
\hline \multicolumn{3}{|c|}{ Mean } & 13.64 & 13.95 & 13.80 \\
\hline \multirow{3}{*}{60} & \multicolumn{2}{|c|}{$S_{0}$} & 12.59 & 12.68 & 12.64 \\
\hline & \multicolumn{2}{|c|}{$\mathbf{S}_{1}$} & 13.78 & 13.84 & 13.81 \\
\hline & \multicolumn{2}{|c|}{$\mathbf{S}_{2}$} & 15.60 & 15.68 & 15.64 \\
\hline \multicolumn{3}{|c|}{ Mean } & 13.99 & 14.07 & 14.03 \\
\hline \multirow{3}{*}{\multicolumn{2}{|c|}{$\begin{array}{l}\text { General mean of } \\
\text { micronutrients + citric acid }\end{array}$}} & $\mathbf{S}_{\mathbf{0}}$ & 11.75 & 11.98 & 11.87 \\
\hline & & $\mathbf{S}_{1}$ & 12.43 & 12.51 & 12.47 \\
\hline & & $\mathbf{S}_{2}$ & 13.22 & 13.32 & 13.27 \\
\hline \multirow[t]{3}{*}{ L. S. D at 0.05} & & $\mathbf{N}$ & 0.57 & 0.54 & 0.36 \\
\hline & & $\mathbf{S}$ & 0.45 & 0.43 & 0.29 \\
\hline & & $\mathbf{N} \times \mathbf{S}$ & 0.90 & 0.71 & 0.52 \\
\hline \multicolumn{3}{|l|}{ C. V \% } & 11.27 & 11.84 & 11.59 \\
\hline
\end{tabular}

$* \mathrm{~S}_{0}=$ Spraying with water, $\mathrm{S}_{1=}$ Spraying with $133 \mathrm{ppm}(\mathrm{Fe}+\mathrm{Zn}+\mathrm{Mn})$ for each element $+1000 \mathrm{ppm}$ citric acid and $\mathrm{S}_{2}=\mathrm{Spraying}$ with $200 \mathrm{ppm}(\mathrm{Fe}+\mathrm{Zn}+\mathrm{Mn})$ for each element $+1500 \mathrm{ppm}$ citric acid.

\section{3. Chemical traits}

\subsubsection{Total chlorophyll of leaves}

Results in Table (8) indicated that the total chlorophyll of leaves at 75 DAS increased significantly with increasing nitrogen rates gradually from 15 up to $60 \mathrm{~kg} \mathrm{~N} \mathrm{fed}{ }^{-1}$. The maximum value for total chlorophyll of leaves was scored from adding $60 \mathrm{~kg} \mathrm{~N}^{\mathrm{N}} \mathrm{fed}^{-1}$. Such results may be due to the fact that nitrogen plays an important role in chlorophyll synthesis. Similar results were found by Mourad (2006) on grain sorghum plants. Regarding the effect of foliar spray of micronutrient mixture in combination with citric acid, Table (8) showed that total chlorophyll of the leaves increased significantly when barley plants were sprayed with $200 \mathrm{ppm}(\mathrm{Fe}+\mathrm{Zn}+\mathrm{Mn})$ for each element plus $1500 \mathrm{ppm}$ citric acid $\left(\mathrm{S}_{2}\right)$. In this respect,
Hanna et al. (2001) found that the total chlorophyll of wheat leaves was increased with spraying 500 or 100 ppm ascorbic acid compared with The control.

Concerning the interaction effect between nitrogen fertilization and foliar spray with micronutrients mixture plus citric acid on total chlorophyll of leaves, the results of Table (8) recorded a significant effect. The maximum value of such trait was scored when plants treated with $60 \mathrm{~kg} \mathrm{~N}^{-1} \mathrm{~d}^{-1}$ and foliar spray by $200 \mathrm{ppm}(\mathrm{Fe}+\mathrm{Zn}+\mathrm{Mn})$ for each element plus $1500 \mathrm{ppm}$ citric acid $\left(\mathrm{S}_{2}\right)$. In this respect, Mourad (2006) reported that the maximum value of total chlorophyll was gained when grain sorghum plants treated with $140 \mathrm{~kg} \mathrm{~N}^{-1} \mathrm{fed}^{-1}$ and sprayed with 500 ppm ascorbic acid plus 500 ppm citric acid. 
Table (8): Total chlorophyll of leaves at $75 \mathrm{DAS}\left(\mathrm{mg} / \mathrm{m}^{2}\right)$ and protein $\%$ of grains of barley as affected by nitrogen fertilization and foliar spray by micronutrients mixture plus citric acid in 2012/2013, 2013/2014 and combined two seasons.

\begin{tabular}{|c|c|c|c|c|c|c|c|}
\hline \multirow[t]{2}{*}{$\begin{array}{c}\text { Nitrogen } \\
\text { level kg N/fed }\end{array}$} & \multirow{2}{*}{$\begin{array}{l}\text { Micronutrients } \\
\text { mixture }+ \\
\text { citric acid } *(\mathbf{S})\end{array}$} & \multicolumn{3}{|c|}{$\begin{array}{c}\text { Total chlorophyll of } \\
\text { leaves at } 75 \text { DAS }\left(\mathrm{mg} / \mathrm{m}^{2}\right)\end{array}$} & \multicolumn{3}{|c|}{ protein $\%$ in grains } \\
\hline & & $\begin{array}{l}2012 / \\
2013\end{array}$ & $\begin{array}{l}2013 / \\
2014\end{array}$ & Comb. & $\begin{array}{l}2012 / \\
2013\end{array}$ & $\begin{array}{l}2013 / \\
2014\end{array}$ & Comb. \\
\hline \multirow{3}{*}{15} & $\mathrm{~S}_{0}$ & 379.83 & 375.93 & 377.88 & 8.30 & 8.21 & 8.26 \\
\hline & $\overline{S_{1}}$ & 395.43 & 388.41 & 391.92 & 8.88 & 8.98 & 8.93 \\
\hline & $\mathrm{S}_{2}$ & 425.33 & 403.08 & 414.21 & 9.02 & 8.97 & 9.00 \\
\hline \multicolumn{2}{|c|}{ Mean } & 400.20 & 389.14 & 394.67 & 8.73 & 8.72 & 8.73 \\
\hline \multirow{3}{*}{30} & $\mathrm{~S}_{0}$ & 394.13 & 389.71 & 391.92 & 9.16 & 9.04 & 9.10 \\
\hline & $\mathrm{S}_{1}$ & 424.55 & 415.97 & 420.26 & 9.82 & 9.88 & 9.85 \\
\hline & $\mathrm{S}_{2}$ & 445.09 & 434.43 & 439.76 & 10.38 & 10.21 & 10.30 \\
\hline \multicolumn{2}{|c|}{ Mean } & 421.26 & 413.37 & 417.32 & 9.79 & 9.71 & 9.75 \\
\hline \multirow{3}{*}{45} & $\mathrm{~S}_{0}$ & 398.29 & 387.21 & 392.75 & 10.14 & 10.23 & 10.19 \\
\hline & $\mathrm{S}_{1}$ & 440.41 & 439.37 & 439.89 & 10.68 & 10.62 & 10.65 \\
\hline & $\mathrm{S}_{2}$ & 473.03 & 463.55 & 468.29 & 11.49 & 11.38 & 11.44 \\
\hline \multicolumn{2}{|c|}{ Mean } & 437.24 & 430.04 & 433.64 & 10.77 & 10.74 & 10.76 \\
\hline \multirow{3}{*}{60} & $\mathrm{~S}_{0}$ & 432.61 & 419.61 & 426.11 & 10.02 & 10.21 & 10.12 \\
\hline & $\mathrm{S}_{1}$ & 478.89 & 474.21 & 476.55 & 10.93 & 11.02 & 10.98 \\
\hline & $\mathrm{S}_{2}$ & 494.23 & 480.45 & 487.34 & 12.14 & 12.08 & 12.11 \\
\hline \multicolumn{2}{|c|}{ Mean } & 468.58 & 458.09 & 463.33 & 11.03 & 11.10 & 11.07 \\
\hline \multirow{3}{*}{$\begin{array}{l}\text { General mean of } \\
\text { micronutrients +citric } \\
\text { acid }\end{array}$} & 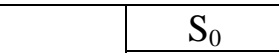 & 401.22 & 393.12 & 397.17 & 9.41 & 9.42 & 9.42 \\
\hline & tric & 434.82 & 429.49 & 432.16 & 10.08 & 10.13 & 10.10 \\
\hline & 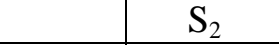 & 459.52 & 445.38 & 452.40 & 10.76 & 10.66 & 10.71 \\
\hline \multirow[t]{3}{*}{ L. S. D at 0.05} & $\mathrm{~N}$ & 7.62 & 7.24 & 4.83 & 0.47 & 0.45 & 0.30 \\
\hline & $S$ & 6.00 & 5.70 & 3.81 & 0.36 & 0.34 & 0.23 \\
\hline & $\mathrm{N} \times \mathrm{S}$ & 11.98 & 11.38 & 7.59 & 0.73 & 0.66 & 0.45 \\
\hline \multicolumn{2}{|l|}{ C. V\% } & 4.93 & 4.31 & 4.62 & 3.76 & 3.54 & 3.65 \\
\hline
\end{tabular}

$* \mathrm{~S}_{0}=$ Spraying with water, $\mathrm{S}_{1=}$ Spraying with $133 \mathrm{ppm}(\mathrm{Fe}+\mathrm{Zn}+\mathrm{Mn})$ for each element $+1000 \mathrm{ppm}$ citric acid and $\mathrm{S}_{2}=$ Spraying with $200 \mathrm{ppm}(\mathrm{Fe}+\mathrm{Zn}+\mathrm{Mn})$ for each element $+1500 \mathrm{ppm}$ citric acid.

\subsubsection{Protein content of grains}

Data of Table (8) show that protein content in grains was increased gradually with raising nitrogen rate from 15 up $60 \mathrm{~kg} \mathrm{~N}$ fed $^{-1}$. Results indicated that treated barley by $60 \mathrm{~kg} \mathrm{~N}$ fed $^{-1}$ significantly increased grain protein content, compared with the other three levels $i . e .15,30$ and $45 \mathrm{~kg} \mathrm{~N} \mathrm{fed}^{-1}$. Such results can be ascribed to the function of nitrogen in plant metabolism viz constituent of amino and nucleic acids, many cofactors and cellular compounds. Similar results were found by Zeidan (2007) and Abd El-Rahman et al. (2012).

Regarding the effect of foliar application with micronutrients mixture and citric acid, results in Table (8) recorded a significant effect on protein content of grains. The maximum value was scored when barley plants were sprayed with $200 \mathrm{ppm}(\mathrm{Fe}+\mathrm{Zn}+\mathrm{Mn})$ for each element plus
1500 ppm citric acid $\left(S_{2}\right)$ compared with $S_{0}$ and $S_{1}$. Such finding may be due to the role of micronutrients on enhancing the enzymes which help in protein formation process (Ghaly et al., 1992). Anton and Bassiem (1998) reported that in peanut, the foliar spray of $500 \mathrm{ppm}$ ascorbic acid recorded the highest value of protein $\%$ in seeds flowed by spraying $500 \mathrm{ppm}$ citric acid.

The interaction effect between nitrogen fertilization and foliar spray with micronutrients mixture plus citric acid recorded a significant effect on protein content of grains. The maximum value of such traits was achieved when plants were treated with $60 \mathrm{~kg} \mathrm{~N}^{-1} \mathrm{~d}^{-1}$ and foliar spray by $200 \mathrm{ppm}$ micronutrients mixture $(\mathrm{Fe}+\mathrm{Zn}+\mathrm{Mn})$ for each element plus $1500 \mathrm{ppm}$ citric acid. These results are in harmony with those reported by Anton et al., (1999). 


\section{Conclusion}

In the light of the present results, the maximum grain yield and protein content in grains of barley were obtained from plants treated by $60 \mathrm{~kg} \mathrm{~N}^{-1}$ and foliar spray with $200 \mathrm{ppm}$ micronutrients mixture $(\mathrm{Fe}+\mathrm{Zn}+\mathrm{Mn})$ for each element plus $1500 \mathrm{ppm}$ citric acid. However, from the economic point of view and nitrogen fertilizer conservation it is advisable to practice $45 \mathrm{~kg} \mathrm{~N}_{\text {fed }}{ }^{-1}$ under calcareous soils condition in Nubaria region, due to insignificant differences in grain yield observed between adding 45 and $60 \mathrm{~kg} \mathrm{~N} \mathrm{fed}^{-1}$.

\section{REFERENCES}

Abdel-Hadi A. H., Hassan M. A. M., Moustafa A. A. and Shalaby S. (1990). Proceeding of the Conference of Soil Fertility and Foliar fertilization., 1, January, 1990 pp 332-345 Egypt.

Abdo Fatma A. and El-Moselhy M. A. (2004). Response of barley plant to foliar spray with ascorbic acid under different levels of nitrogen application. Egypt. J. Appl. Sci. 19 (2) :221-332.

Abdel-Rahman M. F. S., Khalil F. A. F. and Anton N. A. (2012). Response of some barley cultivars to nitrogen sources and rates grown in alkaline sandy soil. Res. J. Agric. and Bio. (Sci., 3(6): 934-938.

Anton N. A., Abdel-Nour A. Sh. and Abdel Aziz E. A. (1999). Response of barley to ascorbic, citric acids and micronutrients mixture under sandy soil conditions. Zagazig J. Agric. Res., 26 (6): 15531563.

Anton N. A. and Bassiem M. M. (1998). Effect of phosphorus and potassium fertilizers and foliar spray with ascorbic and citric acids on peanut plants under sandy soil conditions. Zagazig J. Agric. Res., 25(5): 733-742.

A.O.A.C. (1990). Official Methods Of Analysis, $15^{\text {ed }}$, Association of Official Agricultural Chemists, Washington, D. C., USA.

Dirienzo D. B., Weeb K. E. J., Brann R. D. E. and Alley M. M. (1991). Effect of spring nitrogen application on barley forage yields and silage fermentation. J. Production of Agric. (USA), 4 (1): 3944.

El-Habbal M. S., Osman A. O. and Badram M. M. (1995). Effect of some micronutrient fertilizer and transplanting on wheat productivity in newly reclaimed saline soil. Annals Agric., Ain Shams Univ., Cairo, 40(1): 145-152.

El-Sayed A. A. and Abd El-Hadi (1991). Effect of foliar application of some micronutrients and foliar fertilizers on yield of barley in sandy and calcareous soils. Zagazig J. Agric. Res., 18 (4) pp: 25-37.

Ghaly S., Osman A. O. and Abd El-Aziz I. M. A. (1992). The interaction of some micronutrients and rhizobia on lentil yield grown on sandy soils. Egypt J. Agric. Res., 70 (4): 1025-1035.

Hanna Fardoas R., Abdo Fatma A. and Anton N. A. (2001). Response of wheat plant to foliar application with ascorbic acid, copper and boron. J. Agric. Sci. Mansoura Univ., 26 (10): 5971-5983.

Megahed M. A. (2003). Effect of seeding rates and nitrogen fertilizer levels on newly hull-less barley line under sprinkler irrigation system in poor sandy soils at Ismailia Governorate. Egypt. J. Sci., 18(2): 108-119.

Megahed M. A., Orabi A. M. and Badwy A. A. (1999). Effect of irrigation intervals and nitrogen levels under New valley conditions. Nile valley and Red Sea Regional Program. ICARDA. 11 ${ }^{\text {th }}$ Annual coordination meeting. Egypt, ARC, Cairo 5-9 September 1999. pp: 105-109.

Megahed M. A., Mohamed Samia G. A. and Ali A. M. A. (2001 b). Determination of the important factors of barley yield under different NPK fertilizer regime in new reclaimed soil. Fayoum J. Agric. Res. Dev., 15(2): 125-134.

Miernyk J. A. and Trelease R. N. (1981). Role of malate synthesis in citric acid synthesis by maturing cotton embryos. A Proposal. Plant Physiol., 68: 875-881.

Monge and Bugbe (1992). Inherent limitation of nondestructive chlorophyll meters-A comparison of types meters. Hort. Sci., 27: 69-71.

Mourad A. E. A. A. (2006). Response of grain sorghum to foliar spray with ascorbic and citric acids under different rates of nitrogen fertilizer in sandy soil. J. Agric. Sci. Mansoura Univ., 31(11): 68256837.

Osawa T. (1973). Heavy metal toxicities in vegetable crop. The effect of iron concentration in the nutrient solution on 
Osawa T. (1973). Heavy metal toxicities in vegetable crops. The effect of iron concentration in the nutrient solution on zinc toxicities in vegetable crops. Hort. Abs., 844-848.

Roy D. K. and Singh B. P. (2006). Effect of level and time of nitrogen application with and without vermicompost on yield, yield attributes and quality of malt barley (Hordeum vulgare). Indi J. Agron., 51(1).

Ryan J., Garabet S., Harmsen K. and Rashid A. (1996). A Soil and plant analysis. Manual Adopted for the West Asia and North Africa Region. ICARDA, Aleppo,
Syria, pp. 140-146.

Snedecor George W. and Cochran William G. (1989). Statistical Methods, $8^{\text {th }}$ Ed., Iowa State University Press, USA..

Steel R. G. D. and Torrie J. A. (1980). Principles and procedures of statistics, $2^{\text {th }}$ Ed., Mc Graw Hill Pub. Co., New york, USA. PP. 633.

Watson D. J. (1952). The physiological basis of variation in yield. Adv. Agron., 4: 101145.

Zeidan M. S. (2007). Response of some barley cultivars to nitrogen sources and rates grown in alkaline sandy soil. Res. J. Agric. and Biol. Sci., 3(6): 934-938.

استجابة الثعير للتسميد النتروجينى و الرش الورقى بمخلوط العناصر الصغرى وحامض الستريك

محمد زكريا محمود شندى - هبة على جمعة - مارى عريان ناثد*

قسم بحوث الثعير و *قسم بحوث فسيولوجيا المحاصيلـ معهد بحوث المحاصيل ـ مركز البحوث الزر اعيةـ الجيزة ـ مصر

\section{ملخص}

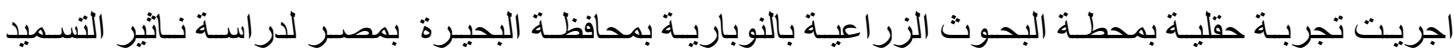

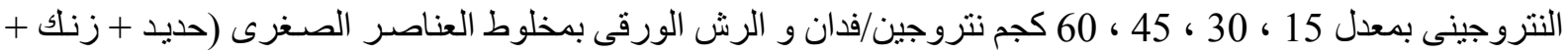

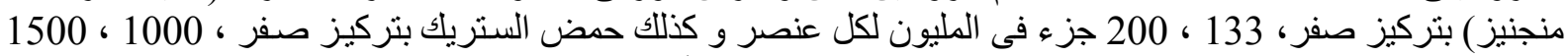

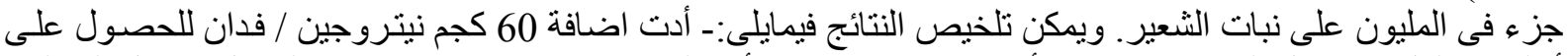

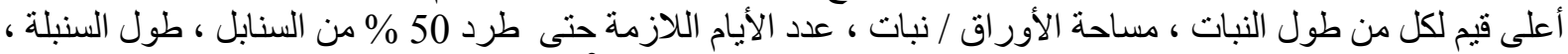

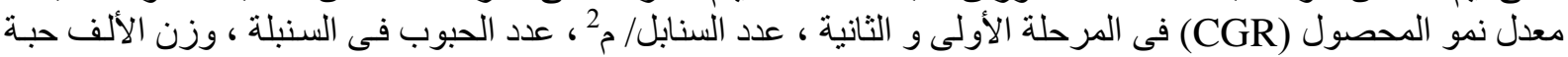

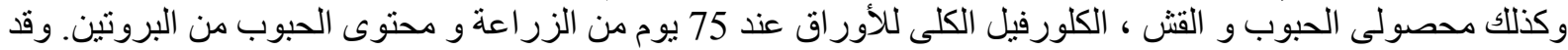

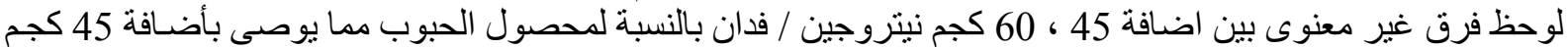

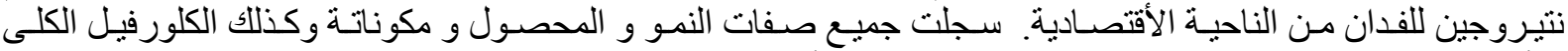

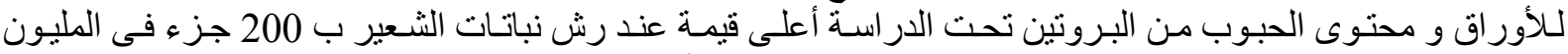

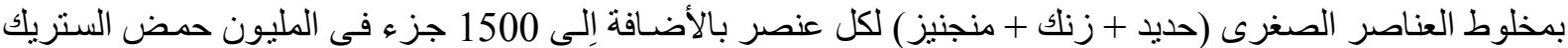

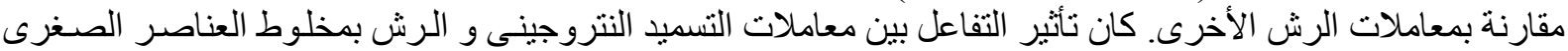

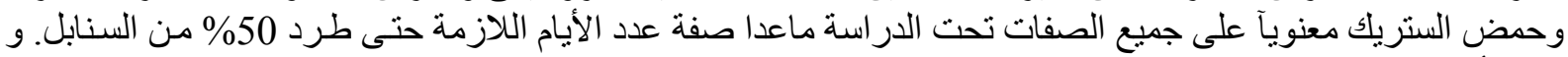

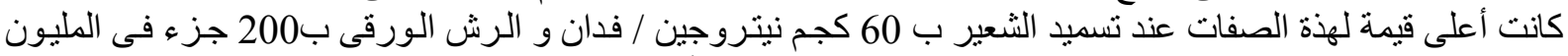

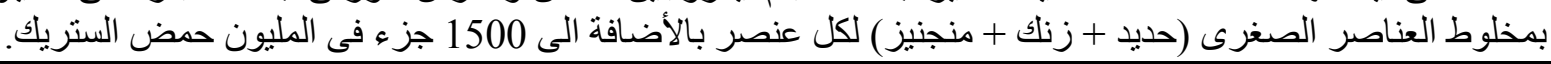

